\title{
Transport du phosphore inorganique par le tubule proximal du rein
}

Le phosphate présent dans le tubule proximal du rein est réabsorbé contre un gradient électrochimique, ce qui implique l'intervention de systèmes de transport actifs dont les mécanismes commencent seulement à être connus.

\section{Michèle G. Brunette Professeur titulaire, département de pédiatrie, université de Mon- tréal.}

\section{RÉFÉRENCES}

I. Freeman D, Bartlett S, Radda G, Ross B. Energetic of $\mathrm{Na}$ transport in the kidney saturation transfer. Biochem Biophys Acta 1983; 762: 32536.

2. Kinne $\mathbf{R}$, Berne $\mathrm{RW}$, Hoffmann $\mathbf{N}$, Murer $\mathrm{H}$ Phosphate transport by isolated renal and intestinal plasma membrancs. Adv Exp Med Biol 1977; 8I : 265-77.

3. Brunette MG, Béliveau R, Chan M. Effect of temperature and $\mathrm{pH}$ on phosphate transport through brush border membrane vesicles in rats. Can 7 Physiol Pharmacol 1984a; 62 : 229-34

4. Brunette MG, Chan M, Maag U, Béliveau R Phosphate uptake by superficial and decp nephron brush border membranes. Effect of dietary phosphate and parathyroid hormonc. Pfugers Arch 1984 b; 400 : 356-62.

5. Baumann K, De Rouffignac C, Roinel N, Rumrich G, Ullrich KJ. Renal phosphate transport: inhomogeneity of local proximal transport rates and sodium dependence. Pfugers Arch 1975; $356: 287-97$.

6. Dennis VW, Woodhall PB, Robinson RR Characteristics of phosphate transport in isolated proximal tubule. Am $\mathcal{F}$ Physiol 1976; 231 : 979-85.

7. Brunette MG, El Mernissi G, Doucet A. Na uptake by renal brush border membrane and $\mathrm{Na}-\mathrm{K}$ ATPase activity along the nephron in genetic hypophosphatemic mice. Can 7 Physiol Pharmacol 1985 (sous presse)

\section{ADRESSE}

M. G. Brunette : Département de Pédiatrie, hôpital Maisonneuve, 5415, boulevard de l'Assomption, $\mathrm{H}_{\mathbf{I}} \mathrm{T} 2 \mathrm{M}_{4}$, Montréal. a plus grande partie de la réabsorption rénale du phosphate inorganique $\left(\mathrm{PO}_{4}\right)$ a lieu dans le tubule proximal, qui est donc le site d'action principal, sinon unique, des facteurs régulateurs de la phosphaturie. La concentration du liquide tubulaire en $\mathrm{PO}_{4}$ étant de $60 \%$ de celle du plasma dans la partie moyenne du tubule proximal, et le potentiel électrique étant seulement d'environ $+\mathrm{I} \mathrm{mV}$ dans ce segment alors que le potentiel intra-cellulaire est très négatif, le transport du $\mathrm{PO}_{4}$ se fait contre un gradient électrochimique et implique donc des mécanismes actifs. Au cours de ce processus, le $\mathrm{PO}_{4}$ a trois étapes cellulaires à franchir : la bordure en brosse, le cytosol et la membrane basolatérale. Le gradient électrochimique étant essentiellement situé de part et d'autre de la bordure en brosse, il est compréhensible que les mécanismes impliqués dans le transport du $\mathrm{PO}_{4}$ par cette membrane aient fait l'objet de nombreux travaux. La bordure en brosse possède, comme nous le verrons, tous les éléments nécessaires pour transporter le $\mathrm{PO}_{4}$ mais ceuxci sont influencés par le métabolisme intra-cellulaire ainsi que par de nombreux facteurs extérieurs à la cellule (figure I).

\section{La bordure en brosse}

Le transport du $\mathrm{PO}_{4}$ par la membrane de la bordure en brosse (MBB) est un mécanisme actif. In vivo, le transport du $\mathrm{PO}_{4}$ par la MBB se fait contre un gradient électrochimique. La concentration du $\mathrm{PO}_{4}$ inorganique libre à l'intérieur de la cellule, telle que déterminée par résonance magnétique nucléaire [I], est semblable à celle du phosphore intraluminal, mais le potentiel électrique $(\mathrm{V})$ à l'intérieur de la cellule est très négatif par rapport à celui de la lumière tubulaire et impose un gradient défavorable à l'entrée du $\mathrm{PO}_{4}$. Cette hypothèse d'un transport actif fut confirmée par les études sur des préparations de membranes in vitro: l'accumulation de $\mathrm{PO}_{4}$ en fonction du temps par les vésicules de MBB ne contenant pas de sodium, incubées dans un milieu contenant du $\mathrm{Na}$ et du phosphate, montre une entrée initiale très rapide du $\mathrm{PO}_{4}$, atteignant une accumulation maximale entre 30 secondes et 2 minutes selon la température de l'incubation. Ce pic de $\mathrm{PO}_{4}$ intra-vésiculaire témoigne d'un mécanisme de transport du $\mathrm{PO}_{4}$ couplé à celui du sodium. Le transport initial augmente avec la concentration de phosphate dans le milieu jusqu'à ce que soit atteint un niveau de saturation, suivant les lois de Michaelis Menten.

Un ou deux systèmes de transport? A des concentrations relativement faibles de $\mathrm{PO}_{4}(0,04$ à 0, I $\mathrm{mM}$ ), les valeurs de $\mathrm{Km}$ (constante de Michaelis Menten) et Vmax (vitesse initiale maximale) sont de $80 \mu \mathrm{M} \mathrm{PO}_{4}$ et $5 \mathrm{nmoles} / \mathrm{Io}$ $\mathrm{sec}^{-1} \cdot \mathrm{mg}^{-1}$ protéines respectivement $[2,3]$. Cependant, avec des concentrations plus élevées de $\mathrm{PO}_{4}$, 
une deuxième composante de transport apparaît, dont la vitesse maximale est supérieure, et l'affinité inférieure, aux valeurs correspondantes du premier système. Ainsi, lorsque le transport du $\mathrm{PO}_{4}$ est étudié en utilisant une large gamme de concentrations et que les valeurs obtenues sont figurées selon la méthode de Eadie-Hof stee (vitesse en fonction de vitesse/substrat), on observe une courbe brisée qui se décompose en deux constituantes dont les $\mathrm{Km}$ respectifs sont $70 \mu \mathrm{M}$ et I, $18 \mathrm{mM}$ [3]. Cette dualité de systèmes de transport cependant n'est mise en évidence qu'à des températures d'incubation supérieures à $20-25^{\circ} \mathrm{C}$, et avec des vésicules provenant du cortex superficiel. Le cortex profond, du moins chez le rat, ne semble posséder que le système de transport à haute affinité [4].

\section{Le rôle du sodium}

L'énergie nécessaire au transport du $\mathrm{PO}_{4}$ par la cellule du tubule proximal, et particulièrement par la $\mathrm{MBB}$, provient du gradient électrochimique de sodium. Si l'on abaisse le gradient extraintracellulaire de sodium dans des expériences de microperf usion in vivo [5], ou de microperfusion de tubule isolé in vitro [6], le transport de $\mathrm{PO}_{4}$ est immédiatement inhibé. De la même façon, nous l'avons vu, l'entrée du $\mathrm{PO}_{4}$ dans les vésicules de MBB préparées in vitro dépend largement du gradient de sodium. Le sodium influence la cinétique du transport de $\mathrm{PO}_{4}$ en changeant l'affinité de la molécule transporteuse pour le $\mathrm{PO}_{4}$ : plus le sodium dans le milieu d'incubation est élevé, plus l'affinité pour le $\mathrm{PO}_{4}$ augmente [7]. Mais le transport du $\mathrm{PO}_{4}$ dépend aussi de l'affinité du système pour le sodium, et cette

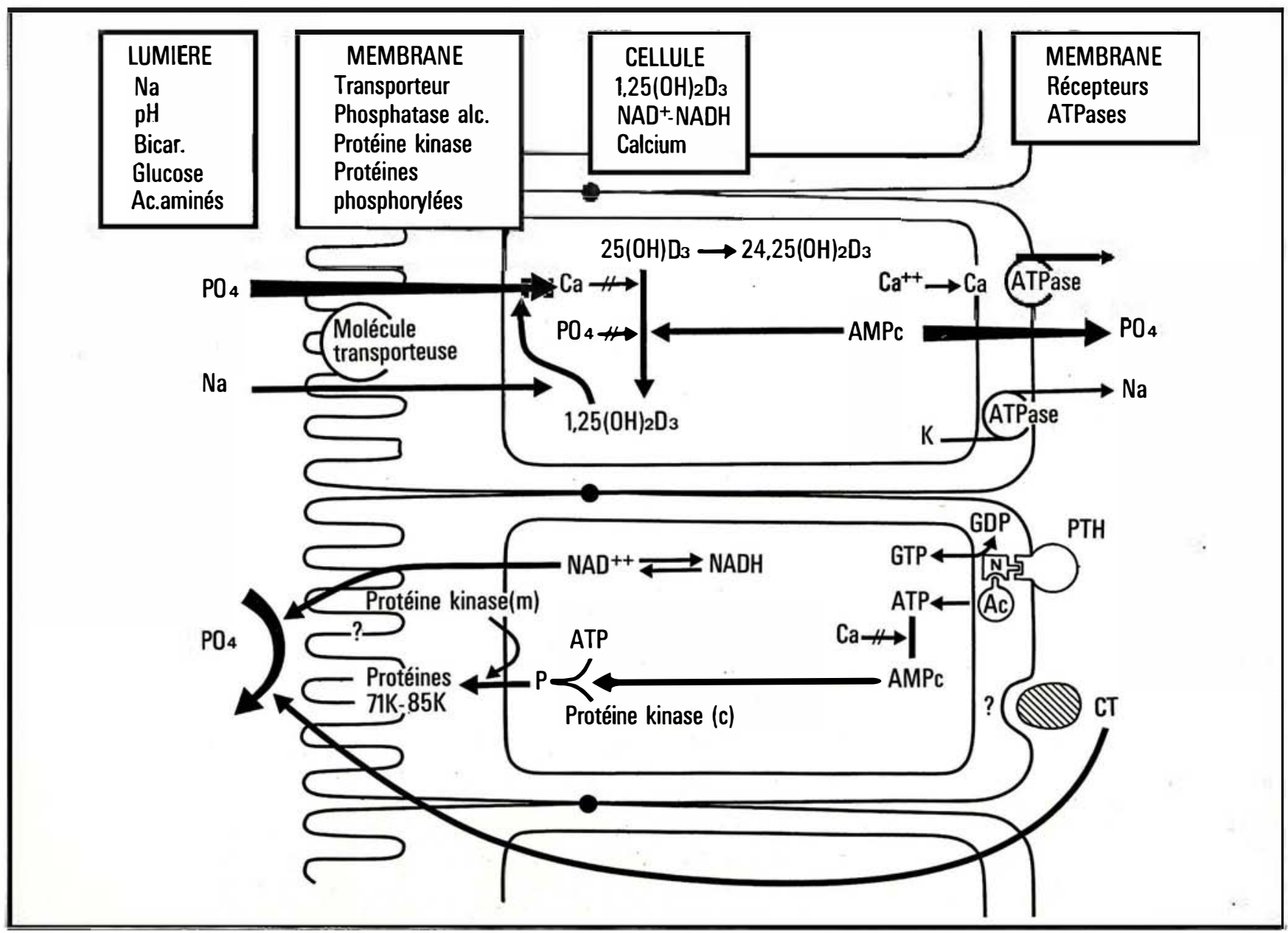

Figure 1. Représentation schématique des mécanismes impliqués dans le transport du phosphore inorganique par la cellule du tubule proximal. Les facteurs sont répartis en quatre catégories selon leur situation topographique : lumière tubulaire, membrane luminale, milieu intracellulaire, et membrane basolatérale. Les flèches indiquent soit le mouvement des substrats, soit la direction des réactions chimiques. Les flèches barrées ( $\$$ ) indiquent que la substance a une action inhibitrice sur la réaction en question.

$A c=$ adénylate cyclase; $C T=$ calcitonine; $(m):$ membranaire; $(c)$ : cytosolique; $N=$ protéine $N ; G T P=g u a n o s i n e ~ t r i p h o s-$ phate; GDP = guanosine diphosphate.

$m / s n^{\circ} 8$, vol. 2, octobre 86 


\section{REFERENCES}

8. Amstutz M, Mohrmann M, Gmaj P, Murer H. Effect of $\mathrm{pH}$ on phosphate transport in rat renal brush border membrane vesicles. $\mathrm{Am}$ 7 Physiol 1985; 248: F 705-10.

9. Cheng L, Sacktor B. Sodium gradient dependent phosphate transport in renal brush border membrane vesicles. 7 Biol Chem 1981; 256: 1 55664

10. Sacktor B, Cheng L. Sodium gradient dependent phosphate transport in renal brush border membrane vesicles. Effect of an intravesicular extravesicular proton gradient. $7 \mathrm{Biol}$ Chem $198 \mathrm{I}$; 256: $8080-4$.

II. Hoffman N, Thees M, Kinne R. Phosphate transport by isolated renal brush border vesicles. Pflugers Arch, 1976; 362: 1 47-56.

12. Burckhardt G, Stern H, Murer H. The influence of $\mathrm{pH}$ on phosphate transport into rat renal brush border membranes vesicles. Pflugers Arch 1981; 390: I 91-7.

13. Plante GE, Petitclerc C, Nawar T, Erian R Increased phosphaturia during inhibition of renal alkaline phosphatase. Miner Electrolyte Metab 1979; 2: 258 .

14. Brunette MG, Dennis VW. Effect of L-bromotetramisole on phosphate transport by the proximal renal tubule: Failure to demonstrate a direct involvement of alkaline phosphatase. Can $7 \mathrm{Phy}$ siol Pharmacol 1982; 60: 276-81.

15. Yusufi ANK, Low MG, Turner ST, Dousa TP. Enzymatic removal of alkaline phosphatase from renal brush border membrane: Effect on $\mathrm{Na}^{+}$-dependent transport of phosphate. The American Society of Nephrology, I6th annual meeting 1983; abstract: 2 I A

I6. Brunette MG, Chan M, Lebrun M. Phosphatase activity along the nephron of mice with hypophosphatemic vitamin D resistant rickets. Kidney Int 1981; 20: 181-7.

17. Kinne R, Shlatz LV, Kinne-Saffran E Schwartz IL. Distribution of membrane bound cyclic AMP dependent protein kinase in plasm membranes of cells of the kidney cortex. 7 Membr Biol 1975; 24: 145-59.

I8. Hammerman MR, Hansen VA, Morrissey JJ Cyclic AMP-dependent protein phosphorylation and dephosphorylation alter phosphate transport in canine renal brush border vesicles. Biochim Biophys Acta 1983; 755 : 10-6.

19. Brunette MG, Allard S. Renal brush border membrane phosphorylation: influence of PTH status and dietary phosphate on the intrinsic protein kinase activity. Can 7 Physiol Pharmacol $1985 ; 63$ (sous presse).

20. Kessler RJ, Fanestil DD. Identification of a phosphate binding proteolipid in kidney brush border. In: Bronner F, Peterlik M, eds. Calcium and Phosphate Transport Across Biomembranes. New York; Academic Press, 1981: 123-6. dernière est elle-même modifiée par le $\mathrm{pH}$ : en milieu acide, cette affinité baisse [8].

Le transport du $\mathrm{PO}_{4}$ dépend du $\mathrm{pH}$. Théoriquement le $\mathrm{pH}$ est susceptible d'influencer le transport du $\mathrm{PO}_{4}$ de deux façons : d'une part, en modifiant la concentration respective des formes mono et divalentes de l'ion phosphate et d'autre part, en agissant directement sur la molécule transporteuse. Les études effectuées sur vésicules mesurent le transport du $\mathrm{PO}_{4}$ total. Il n'est pas possible d'étudier directement avec cette technique le transport de chacune des formes séparément (en dehors de $\mathrm{pH}$ extrêmes). Des arguments indirects cependant suggèrent que c'est la forme divalente qui est transportée. En effet, quel que soit le $\mathrm{pH}$, l'inverse du transport de $\mathrm{PO}_{4}$ est en relation directe avec l'inverse du carré de la concentration en sodium, comme si deux molécules de sodium accompagnaient une molécule de $\mathrm{PO}_{4}$. Par ailleurs, Cheng et Sacktor [9] ont incubé des vésicules dans des milieux à $\mathrm{pH}$ variables mais composés de telle sorte que les formes mono ou divalentes demeurent constantes. Lorsque les monophosphates sont constants et que les diphosphates augmentent (donc le $\mathrm{pH}$ aussi), le transport total augmente. Cependant, lorsque les diphosphates restent constants, et que le $\mathrm{pH}$ augmente, le transport augmente quand même. Il semble donc que, non seulement la forme divalente est préférentiellement transportée, mais qu'un $\mathrm{pH}$ relativement alcalin stimule en soi le transporteur.

Récemment, nous avons confirmé cet effet en étudiant les cinétiques de transport du $\mathrm{PO}_{4}$ par des $\mathrm{MBB}$ à différents $\mathrm{pH}$. Nous avons trouvé que l'augmentation du $\mathrm{pH}$ abaisse la $\mathrm{Km}$ du système à forte affinité. Si nos données sont analysées en utilisant l'hypothèse que seule la forme divalente du phosphate est transportée, les cinétiques se réorganisent pour ne donner qu'une seule Km et plusieurs Vmax, comme si la forme divalente était transportée avec une même affinité quel que soit le $\mathrm{pH}$, mais que ce dernier influençait directement la Vmax du transporteur [3]. Dans cette étude, le pH optimum était de 7,5 .
Enfin, le pH à l'intérieur des vésicules semble aussi avoir de l'importance : un $\mathrm{pH}$ acide, dans des conditions d'équilibre de sodium (sodium intérieur = sodium extérieur) semble favoriser le transport de $\mathrm{PO}_{4}$ [Io].

Le transport du $\mathrm{PO}_{4}$ est-il ou n'est-il pas électrogénique? Dans leur première étude sur les $\mathrm{MBB}$, Hoffman et coll. $[I I]$ ont étudié l'effet positif du $\mathrm{NaCl}$ dans le milieu d'incubation sur le transport de $\mathrm{PO}_{4}$ et ils en ont conclu que le gradient de $\mathrm{NaCl}$ entraînait un potentiel de membrane négatif à l'intérieur des vésicules. Le remplacement de l'ion $\mathrm{Cl}^{-}$par des ions $\mathrm{SCN}^{-}$plus diffusibles, ou par du cyclamate qui dépolarise la membrane, accentue et décroît respectivement le transport, confirmant cette hypothèse. Il semble donc qu'une certaine partie du mouvement du $\mathrm{PO}_{4}$ à travers la membrane s'accompagne d'un transport de charges positives. Hoffman suggérait alors que ce transport de charges pouvait être dû au transport du $\mathrm{PO}_{4}$ monovalent avec $2 \mathrm{Na}^{+}$. En $\mathrm{I} 98 \mathrm{I}$, Burckhardt $e t$ coll. [12] reprennent cette idée et étudient le changement du potentiel électrique intravésiculaire lors du transport de $\mathrm{PO}_{4}$, à l'aide d'indicateurs fluorescents. Lorsque du $\mathrm{PO}_{4}$ est ajouté au milieu d'incubation, ils observent une différence de potentiel électrique positif à l'intérieur des vésicules, comme si deux ions $\mathrm{Na}$ étaient transportés tant avec les formes monovalentes que divalentes de $\mathrm{PO}_{4}$. Pourtant, l'induction d'un potentiel intravésiculaire négatif par le truchement d'un potentiel de diffusion de potassium (intérieur > extérieur) induit par de la valinomycine [1 2] ou d'un potentiel de diffusion de protons [9, Io], n'influence pas le transport du $\mathrm{PO}_{4}$. On ne sait donc pas encore de façon certaine si ce transport est électrogénique ou non.

Le rôle de la phosphatase alcaline dans le transport du $\mathbf{P O}_{4}$ par la MBB est obscur. Il a été proposé que la phosphatase, une enzyme largement présente dans les $\mathrm{MBB}$, joue un rôle important dans le transport du $\mathrm{PO}_{4}$. La perfusion in vivo de lévamisole, un inhibiteur spécifique de cette enzyme, dans l'artère rénale entraîne une phos- 
phaturie du même côté [13]. Cependant, il fut difficile d'établir une corrélation étroite entre transport de $\mathrm{PO}_{4}$ par la $\mathrm{MBB}$ et son contenu en phosphatase alcaline. L'inhibition de l'activité de l'enzyme par un analogue du lévamisole, le tétramisole, ne change pas le transport de $\mathrm{PO}_{4}$ par des vésicules de MBB [14]. De plus, des expériences qui consistent à enlever l'enzyme de la membrane par des procédés chimiques réussirent à démontrer que l'intégrité du transport n'était pas liée à la présence de la phosphatase alcaline [15]. Il reste tout de même vrai que cette enzyme est localisée très précisément dans le segment du néphron qui transporte le phosphore, particulièrement là où ce transport est maximal, c'est-à-dire au début du tubule proximal, et qu'elle est abaissée dans la maladie génétique dont le principal trait est un défaut de transport du $\mathrm{PO}_{4}$ [I6]. Le substrat naturel de la phosphatase alcaline est inconnu. Son identification pourrait éventuellement aider à comprendre le rôle de cette enzyme mystérieuse dont le $\mathrm{pH}$ optimal d'activité est loin d'être physiologique.

La MBB une fois séparée de la cellule, se souvient des activités de transport qu'elle avait in vivo.

Les vésicules de MBB provenant d'animaux ayant été carencés en $\mathrm{PO}_{4}$, parathyroïdectomisés ou surchargés en hormone de croissance, ont une capacité accrue de transport de $\mathrm{PO}_{4}$, en comparaison de celles des animaux témoins et inversement dans les situations opposées. On ignore la nature des changements responsables de cette " mémoire membranaire ". Comme certains de ces facteurs agissent très rapidement, en moins d'une heure, il est probable qu'ils ne modifient pas la synthèse du transporteur, mais plutôt qu'ils changent topographiquement ou biochimiquement la disponibilité des molécules transporteuses existantes dans la membrane.

On sait depuis longtemps que l'action phosphaturique de l'hormone parathyroidienne s'accompagne in vivo d'une phosphorylation de certaines protéines de la MBB [1 7]. Cette phosphorylation implique
l'AMP cyclique formé sous la dépendance de l'hormone ainsi que des protéines kinases dont une au moins est située dans la membrane elle-même. La déphosphorylation se fait par une phosphatase, elle aussi située dans la membrane. La phosphorylation in vitro de la MBB avec de l'ATP exogène et la protéine kinase endogène s'accompagne d'une baisse de transport de $\mathrm{PO}_{4}$ par les vésicules correspondantes. Inversement, la déphosphorylation de cette même membrane rétablit le transport [18]. Ces observations suggèrent, sans le prouver, l'existence d'un lien direct entre phosphorylation membranaire et transport du $\mathrm{PO}_{4}$. Cependant, on ne sait pas si cette phosphorylation est responsable du phénomène de "mémoire membranaire" induit par l'action de l'hormone parathyroïdienne, et encore moins si elle intervient dans les modifications de la MBB liées au contenu du régime alimentaire en $\mathrm{PO}_{4}$.

Afin de vérifier de façon indirecte si l'hormone parathyroïdienne et le régime modifient la MBB de façon analogue, nous avons étudié la capacité des protéines membranaires à être phosphorylées in vitro par la protéine kinase de la membrane elle-même chez des animaux dans différentes conditions diététiques et hormonales. Nous avons mis en évidence au moins deux protéines $(\mathrm{Mr}=7 \mathrm{I} \mathrm{K}$ et $84 \mathrm{~K})$ dont la phosphorylation in vitro est dépendante de l'AMP cyclique. Aucun des états physiologiques étudiés n'influence de façon significative la phosphorylation maximale des deux protéines en question : la Vmax de cette phosphorylation, lorsque diverses concentrations d'ATP sont utilisées, est tout à fait semblable chez tous les animaux, indépendamment du régime ou de l'activité de l'hormone parathyroïdienne (PTH) [I9].

Mais quelle est cette molécule transporteuse? Les bases moléculaires du transport du $\mathrm{PO}_{4}$ demeurent inconnues. Les études qui ont cherché à les identifier se sont heurtées à l'absence de marqueur spécifique. Alors que la molécule qui transporte le glucose lie de façon compétitive la phlorizine, aucune substance connue ne se lie de façon semblable au transpor- teur de phosphore. En revanche, plusieurs protéines de la MBB lient le $\mathrm{PO}_{4}$ avec une grande affinité. En dehors de la phosphatase alcaline qui, comme nous l'avons vu, ne semble pas jouer un rôle déterminant dans le transport du $\mathrm{PO}_{4}$, Kessler et Fanestil [20] ont décrit et isolé à partir de MBB de rein de lapin un protéolipide, la phosphorine, qui lie le $\mathrm{PO}_{4}$ inorganique de façon spécifique et avec une forte affinité. Cette liaison est inhibée par certains inhibiteurs du transport de $\mathrm{PO}_{4}$, et elle est influencée par la présence d'EDTA, comme l'est, dans une certaine mesure, le transport de $\mathrm{PO}_{4}[2 \mathrm{I}]$. Ces auteurs suggèrent que ce protéolipide est impliqué dans le transport de $\mathrm{PO}_{4}$.

\section{Le cytosol}

Nos connaissances sur le sort du $\mathrm{PO}_{4}$ au cours de son transport intracellulaire sont très restreintes.

Concentration cytosolique de phosphates. La concentration de phosphore inorganique dans la cellule a été évaluée, entre autre, par résonance magnétique nucléaire. Freeman et coll. [I] rapportent des valeurs de $2,4 \mu \mathrm{mol} / \mathrm{gm}$ de tissu, ce qui revient approximativement à I $\mathrm{mmol} /$ litre d'eau intracellulaire. Le $\mathrm{PO}_{4}$ intramitochondrial n'étant probablement pas détecté par la technique de résonance magnétique à cause de la forte densité de la mitochondrie, il s'agit donc de la concentration cytosolique approximative.

Échange mitochondrial: Une fois entré dans la cellule, le $\mathrm{PO}_{4}$ inorganique est en échange constant avec le $\mathrm{PO}_{4}$ organique, et particulièrement avec les composés phosphorylés à haute énergie (ATP-ADP). Il est aussi transporté par les membranes mitochondriales de façon active, selon deux systèmes, l'un assurant un cotransport phosphore $^{-} /$proton $^{+}$, l'autre assurant l'échange phosphore ${ }^{2-} /$ dicarboxylate ${ }^{2-}$ [22].

Le transport du $\mathrm{PO}_{4}$ par la membrane de la mitochondrie est intimement lié au métabolisme de celle-ci. $\mathrm{Si}$ des tubules isolés sont perfusés dès le début de leur préparation avec un perfusat ne contenant pas de $\mathrm{PO}_{4}$, une réduction du transport 
REFEERENCES

21. Kessler RJ, Vaughn DA. Divalent metal is required for both phosphate transport and phosphate binding to phosphorin, a proteolipid isolated from brush border membrane vesicles. $7 \mathrm{Biol}$ Chem 1 984; 259: 9059-63.

22. Pedersen PL, Wehrle JP. Phosphate transport processes of animal cells. In: Martonosi A, ed. Membrane and Transport, volume I. New York: Plenum Press, 1982: 645-63.

23. Dennis VW, Brazy PC. Divalent anion transport in isolated renal tubules. Kidney Int 1982; 22: 498-506.

24. Gullans SR, Brazy PC, Dennis VW, Mandel LJ. Metabolic inhibitors: effects on metabolism and transport in the proximal tube. Am $\mathcal{F}$ Physiol 1982; 243: F $133-40$

25. Braun-Werness JL, Jackson BA, Werness PG, Dousa TP. Binding of nicotinamide adenine dinucleotide by the renal brush border membrane from rat kidney cortex. Biochim Biophys Acta 1983; 732: 553-61.

26. Kempson SA, Colon-Otero G, Lise-Ou SY, Turner ST. Possible role of nicotinamide adenosine dinucleotide as an intracellular regulator or renal transport of phosphate in the rat. $7 \mathrm{Clin}$ Invest $1981 ; 67$ : 1347-60.

27. Lavender AR, Pullman TN. Changes in inorganic phosphate excretion induced by renal arterial inf usion of calcium. Am 7 Physiol 1963; 205: 1025-32.

28. Oberleithner H, Lang F, Greger R, Sporer $\mathrm{H}$. Influence of calcium and ionophore 23187 on tubular phosphate reabsorption. Pfugers Arch 1979; 379: 37-4I.

29. Ullrich KJ, Rumrich G, Klöss S. Phosphate transport in the proximal convolution of the rat kidncy. II Effect of extracellular $\mathrm{Ca}^{2+}$ and application of the $\mathrm{Ca}^{2+}$ ionophore $\mathrm{A}_{23187}$ in chronic PTX animals. Pflugers Arch 1978; 377: 33-42.

30. Brunette MG, Chan M, Ferrière C, Roberts $\mathrm{KD}$. Site of $1,25(\mathrm{OH})_{2}$ vitamin $\mathrm{D}_{3}$ synthesis in the kidney. Nature 1978; 276: 287-9.

31. Gekle D, Stroder J, Rostoc K. The effect of vitamin $D$ on renal inorganic phosphate reabsorption of normal rats, parathyroidectomized rats and rats with rickets: Pediatr Res 197 I; 5: 40-52.

32. Tsutsumi M, Alvarez U, Avioli LV, Hruska KA. Eff ect of 1,25 dihydroxyvitamin $\mathrm{D}_{3}$ on phospholipid composition of rat renal brush border membrane. Am 7 Physiol 1983; 249: F 11 7-23.

33. Schwab JJ, Klahr S, Hammerman MR. $\mathrm{Na}^{+}$ gradient dependent $\mathrm{Pi}$ uptake in basolateral membrane vesicles from dog kidney. Am $7 \mathrm{Phy}$ siol 1984; 246: F 663-9.

34. Robdell M, Birnbaumer L, Pohl SL, Kraus HMJ. The glucagon-sensitive adenyl cyclase system in plasma membrane of rat liver. V. An obligatory role of guanyl nucleotides in glucagon action. 7 Bio.' Chem 1971; 250: 5826-34. de sodium par la cellule entière est observée. Cet effet fut attribué au fait que l'entrée de plusieurs substrats dans la mitochondrie est dépendante du $\mathrm{PO}_{4}$ [23]. Privée de substrats, la mitochondrie n'assume plus adéquatement ses fonctions métaboliques et énergétiques. De façon analogue, la réduction progressive du métabolisme de la mitochondrie par des agents découplants tels que le CCCP (mchroro-carbonyl-cyanide phénylhydrazone) ou bien par des inhibiteurs de transport d'électrons (roténone) entraîne une baisse proportionnelle du transport du sodium et du $\mathrm{PO}_{4}$, alors que celui du glucose est moins influencé par cette manœuvre. Parallèlement, on observe une réduction de l'ATP intracellulaire [24]. Il semble donc que si le $\mathrm{PO}_{4}$ intracellulaire joue un rôle important dans le métabolisme de la mitochondrie, ce dernier influence aussi la réabsorption du sodium et du $\mathrm{PO}_{4}$. Il est donc tout à fait concevable qu'une anomalie, soit du transport au niveau de la membrane mitochondriale, soit du métabolisme de la mitochondrie elle-même, aboutisse finalement à une phosphaturie anormale.

Influence du $\mathrm{NAD}^{+}$. Parmi les autres facteurs intracellulaires exerçant une influence sur le transport du $\mathrm{PO}_{4}$, le $\mathrm{NAD}^{+}$s'est vu attribuer, ces dernières années, un rôle important. Le $\mathrm{NAD}^{+}$est un nucléotide servant de coenzyme à plusieurs déshydrogénases dans de nombreuses réactions d'oxydoréduction du métabolisme intermédiaire. De plus, le NAD ${ }^{+}$est relié à la ribolysation de protéines membranaires par l'ADP. Kempson, Dousa et leurs collaborateurs ont testé l'hypothèse que le niveau cytosolique de $\mathrm{NAD}^{+}$influence directement le wansport du $\mathrm{PO}_{4}$ par la MBB. Ils ont rapporté que le NAD se liait de façon spécifique sur la MBB à deux sites d'affinité différente [25]. De plus, l'injection de nicotinamide chez le rat entraîne parallèlement une augmentation du contenu en $\mathrm{NAD}^{+}$dans le cortex rénal, une augmentation de la phosphaturie et une diminution du transport de $\mathrm{PO}_{4}$ par les $\mathrm{MBB}$ de ces animaux [26]. Il se peut donc que le $\mathrm{NAD}^{+}$, dont la concentration varie avec les états nutritionnels et hormonaux, joue un rôle de messager important dans la régulation de la phosphaturie.

Le calcium intracellulaire. Le calcium intracellulaire et extracellulaire joue probablement un rôle important, quoique mal défini, dans le transport du $\mathrm{PO}_{4}$. La perfusion dans une artère rénale d'une solution riche en calcium stimule la réabsorption du phosphore $\mathrm{du}$ même côté [27]. De même, l'application d'une solution riche en calcium sur la surface du rein [28] ou dans le capillaire péritubulaire [29] augmente le transport du $\mathrm{PO}_{4}$ par les tubules sous-jacents. Enfin, la microperfusion de l'ionophore 23I87, qui accroît la perméabilité des membranes cellulaires au calcium, stimule la réabsorption du $\mathrm{PO}_{4}$ par le tubule microperfusé [28]. Le calcium libre pourrait intervenir dans le transport du $\mathrm{PO}_{4}$ par son action antagoniste de l'adénylate cyclase responsable de la formation d'AMP cyclique.

Enfin, la cellule du tubule proximal est le site de synthèse de la I,25 $(\mathrm{OH})_{2}$ vitamine $\mathrm{D}_{3}$ [30]. Lorsque la vitamine $D$ ou ses métabolites sont administrés à un rat de façon aiguë, ils augmentent la réabsorption du $\mathrm{PO}_{4}$ filtré [3I]. Comme l'administration de $25 \mathrm{OH}$ vitamine $\mathrm{D}_{3}$ s'accompagne d'une baisse d'excrétion de l'AMP cyclique et que le calcium intracellulaire inhibe l'adénylate cyclase, l'hypothèse a été émise que l'action antiphosphaturique de la I,25 $(\mathrm{OH})_{2} \quad \mathrm{D}_{3}$ s'effectue par une augmentation de la concentration $\mathrm{du}$ calcium intracellulaire. Il demeure aussi possible que cette hormone modifie le transport du $\mathrm{PO}_{4}$ en changeant la composition en phospholipides de la MBB [32].

\section{La membrane basolatérale}

La concentration intracellulaire de $\mathrm{PO}_{4}$ inorganique étant du même ordre que celle du liquide extracellulaire et le potentiel électrique dans la cellule étant négatif, il est généralement admis que l'efflux de cet anion par la membrane basolatérale est passif. Dans leur étude sur des vésicules de membranes basolatéra- 
les, Hoffman et coll. [I I] avaient rapporté que le transport de $\mathrm{PO}_{4}$ présentait une certaine dépendance du Na. Cette dépendance avait été attribuée par les auteurs à une contamination de leur préparation avec des vésicules de bordure en brosse. Pourtant, plusieurs années après, Schwab et coll. [33] confirmaient que le transport de $\mathrm{PO}_{4}$ par la membrane basolatérale nécessitait la présence d'un gradient de $\mathrm{Na}$ tout comme au niveau de la bordure en brosse; mais, à la différence de celui-ci, ce transport était très nettement électrogénique car il augmentait lorsqu'un potentiel électronégatif était généré à l'intérieur des vésicules. Les auteurs ne précisent pas dans leur étude la proportion de vésicules orientées "à l'endroit " et "à l'envers", si bien qu'on ne sait pas si le mouvement de $\mathrm{PO}_{4}$ qu'ils ont étudié correspond à celui qui serait dirigé de façon prédominante vers l'intérieur ou bien vers l'extérieur de la cellule.

\section{Mécanismes régulateurs}

Les deux principaux mécanismes régulateurs de ce transport sont l'hormone parathyroïdienne et le contenu du régime en phosphore.

Action de l'hormone parathyroïdienne (PTH). La PTH est une hormone fortement phosphaturique. Une parathyroïdectomie entraîne donc une rétention aiguë de phosphore. Cette hormone est distribuée uniformément à toutes les cellules de l'organisme. Les cellules cibles du rein contiennent des récepteurs spécialisés qui lient la molécule et servent de médiateur à son action. La membrane basolatérale du tubule proximal contient de ces récepteurs, capables de déclencher une série d'événements aboutissant à une régulation du transport de $\mathrm{PO}_{4}$ par la MBB. L'interaction hormone-récepteur dans la membrane basolatérale active le système adénylate cyclase situé sur le côté interne de cette membrane. L'activation de l'adénylate cyclase implique une protéine membranaire, qui est en quelque sorte régulatrice de cette activation. Cette protéine, appelée N par Robdell [34], et " $G$ unit " par Spiegel [35] lie la guanosine triphosphate (GTP) et la guanosine diphosphate (GDP). L'interaction de l'hormone avec les récepteurs affecte cette protéine de façon telle qu'elle lie préférentiellement la GTP et relâche la GDP. Seul le complexe protéine-GTP active l'adénylate cyclase. Par ailleurs ce complexe décroît l'affinité du récepteur pour l'hormone, ce qui a pour effet d'induire une certaine résistance à l'hormone à la suite d'une stimulation persistante.

Dans le tubule proximal, du moins chez le lapin et la souris, seule la PTH stimule la formation d'AMP cyclique. Le calcium intracellulaire, qui agit probablement par l'intermédiaire de la calmoduline, inhibe l'activité de l'adénylate cyclase. La séquence des événements qui font suite à la liaison de la PTH implique toute la cellule d'un pôle à l'autre. L'adénylate cyclase, une fois activée, stimule la conversion de l'adénosine triphosphate (ATP) en adénosine $3^{\prime} 5^{\prime}$ monophosphate (AMPc) qui sert de second messager à l'hormone. A son tour, l'AMPc active une ou des protéines kinases à la fois dans le cytosol et dans la bordure en brosse [I 7, 36]. Pour effectuer cette activation, l'AMPc doit donc migrer de la membrane basolatérale vers le pôle apical. Les mécanismes impliqués dans cette migration sont inconnus. Les protéines kinases phosphorylent finalement certaines des protéines membranaires à partir de l'ATP cytosolique. Cette phosphorylation engendre probablement des modifications dans la conformation de ces protéines. Mais, comme nous l'avons vu, le lien qui existe entre ces changements et l'effet physiologique (qui est une baisse de transport du $\mathrm{PO}_{4}$ par la bordure en brosse) est loin d'être clair. Cette modification est assez stable cependant puisqu'elle persiste dans la membrane après sa séparation du reste de la cellule, reproduisant in vitro la baisse de capacité de transport qu'elle présentait in vivo.

Mécanisme d'action du phosphore alimentaire. La restriction en phosphore dans le régime alimentaire stimule plus que tout autre facteur, et de façon très spécifique, le transport tubulaire du $\mathrm{PO}_{4}$. En quelques heures, celui-ci est accru, et en quelques jours, la phosphaturie tombe à des niveaux négligeables. Cette action du régime est tellement puissante que, lors d'une déplétion en $\mathrm{PO}_{4}$, elle prévient l'effet phosphaturique de la PTH [37]. Pourtant, la formation d'AMPc est normale dans cette circonstance [37]. L'administration de $1,25(\mathrm{OH})_{2} \mathrm{D}_{3}$ restaure la réponse à la PTH probablement en remédiant, au moins partiellement, à la déplétion en phosphore. Les mécanismes expliquant l'effet majeur du régime sur le transport du $\mathrm{PO}_{4}$ sont encore moins connus que ceux impliqués dans l'action de l'hormone parathyroïdienne. Comme la restriction en phosphore s'accompagne d'une calciurie accrue et d'une baisse de l'excrétion d'AMPc, il a été postulé qu'une baisse de la sécrétion de $\mathrm{PTH}$ pouvait être à l'origine de la rétention de $\mathrm{PO}_{4}$ par le rein [38]. Ce facteur ne saurait rendre compte seul de l'effet du régime puisque, d'une part, un animal parathyroidectomisé nourri avec un régime pauvre en phosphore excrète moins de $\mathrm{PO}_{4}$ que son homologue nourri normalement et que, d'autre part, une surcharge en PTH ne rétablit pas la phosphaturie.

La recherche d'une modification du métabolisme intracellulaire expliquant la rétention de $\mathrm{PO}_{4}$ en cas de carence alimentaire ne fut pas fructueuse. La baisse de l'ATP intracellulaire ne survient que très lentement après plusieurs semaines de carence [38]. La gluconéogenèse rénale tend à être diminuée, ce qui devrait avoir davantage pour effet d'abaisser plutôt que d'augmenter le transport de $\mathrm{PO}_{4}$ [23]. Il est possible que la i $\alpha$ hydroxylase qui est l'enzyme responsable de la synthèse du métabolite actif de la vitamine $\mathrm{D}$, la $\mathrm{I}, 25(\mathrm{OH})_{2} \mathrm{D}_{3}$, dont l'activité est stimulée par la carence en phosphore, ait un rôle à jouer dans la réponse tubulaire au régime pauvre en phosphore. Comme nous l'avons vu, l'administration aiguë de I,25 $(\mathrm{OH})_{2} \quad \mathrm{D}_{3}$ entraîne une augmentation du transport de $\mathrm{PO}_{4}$ dans ce même segment. Mais cette observation ne veut pas nécessairement dire qu'il s'agit du mécanisme responsable de l'influence de l'apport diététique de phosphore. 
Enfin, la bordure en brosse est modifiée par le régime carencé en

\section{REFERENCES}

35. Spiegel AM, Levine MA, Aurbach GD, et al. Deficiency of hormone receptor adenylate cyclase coupling protein: basis for hormone resistance in pseudohypoparathyroidism. Am 7 Physiol 1982; 243: E 37-42.

36. Nimmo HG, Cohen P. Hormonal control of protein phosphorylation. Adv Cyclic Nucleotide Res 1977; 8: 145-266.

37. Steele TH. Renal resistance to parathyroid hormone during phosphorus deprivation. 7 Clin Invest 1976; 58: 1461-4.

38. Kreusser WJ, Kurokawa K, Aznar E, Massry SG. Phosphate depletion. Eff ect on renal inorganic phosphorus and adenine nucleotides urinary phosphate and calcium, and calcium balance Miner Electrolyte Metab 1978; 1: 30-42.

39. Gekle D, Kossmann K. Der Enfluss von thyreocalcitonin auf die phosphatreabsorption der Niere (Mikropunktionsuntersuchunger). Monatsschr Kinderheilkd 1968; 116: 308-10.

40. Berndt TJ, Knox F G. Proximal tubule site of inhibition of phosphate reabsorption by calcitonin. Am 7 Physiol 1984; 246: F 927-30.

4I. Chabardes D, Imbert-Teboul M, Montegut M, Clique A, Morel F. Distribution of calcitonin sensitive adenylate cyclase activity along the rabbit kidney tubule. Proc Natl Acad Sci USA 1976; 73: 3608-12.

42. Oberleithner H, Lang F, Greger R, Sporer H. Additivity of the phosphaturic action of parathyrin and calcitonin in the rat kidney. In: Massry SG, Ritz E, Jahn H, eds. Advances in experimental medicine and biology. Phosphate and minerals in health and disease. New York: Plenum Press, 1980: 1 29-34.

43. Kurokawa K, Nagata N, Sasaki M, Nakane $K$. Effects of calcitonin on the concentration of cyclic adenosine $3^{\prime} 5^{\prime}$ monophosphate in rat kidney in vivo and in vitro. Endocrinology 1974; 94: 1514-18.

\section{TIRES A PART}

M. G. Brunette : Département de Pédiatrie hôpital Maisonneuve, 5415, boulevard de l'As428 l'hormone de croissance et l'insuline $\mathrm{PO}_{4}$ puisque, même après sa séparation du reste de la cellule, elle transporte davantage de $\mathrm{PO}_{4}$ que celle d'un animal témoin. La phosphatase alcaline s'est vue attribuer un rôle dans cette adaptation. En effet, la déplétion en phosphore s'accompagne, à long terme, d'une augmentation de l'activité de cette enzyme [14]. Cette augmentation, cependant, est plus tardive que la réponse tubulaire au régime et, finalement, comme nous l'avons déjà mentionné, il n'y a pas de corrélation étroite entre les deux événements [I5].

\section{Summary}

The renal handling of phosphate has recently received much attention, particularly at the molecular level. Phosphate moves through the brush border membranes (BBM) against a chemical and electrical gradient, which necessitates a source of energy. This transport is coupled with sodium, which moves passively down its chemical and electrical gradient. The hypothesis of a secondary active transport of phosphate has been confirmed by experiments using purified membrane vesicles.

Les autres hormones. Plusieurs autres hormones ont une action sur le transport du $\mathrm{PO}_{4}$ par la cellule du tubule proximal, mais à un degré moindre. La calcitonine exerce une action phosphaturique [39] lorsqu'elle est administrée de façon aiguë. Son mode d'action n'est pas clair car si elle freine la réabsorption du $\mathrm{PO}_{4}$ dans le tubule proximal [39, 40], son action sur l'adénylate cyclase à ce niveau est pratiquement nulle du moins chez le lapin et chez la souris [4I]. Comme, par ailleurs, l'effet phosphaturique de la calcitonine [42], et non l'effet sur l'AMPc [43], est additif à celui de l'action maximale de l'hormone parathyroïdienne, il semble que les mécanismes impliqués dans cette action hormonale soient indépendants du système AMP cyclique. Enfin, plusieurs autres facteurs, d'importance moindre que ceux déjà mentionnés, influencent le transport du $\mathrm{PO}_{4} \mathrm{par}$ le tubule proximal, comme qui le stimulent légèrement.

\section{Conc/usion}

Même si ces dernières années améliorèrent nos connaissances sur les différentes étapes du transport du $\mathrm{PO}_{4}$ par la cellule tubulaire proximale, beaucoup d'inconnues persistent quant à la nature biochimique et physicochimique de chacune de ces étapes; et c'est probablement pour cette raison que les mécanismes pathogéniques impliqués dans les maladies congénitales de la réabsorption du $\mathrm{PO}_{4}$ ne sont pas encore élucidés
Phosphate uptake is modulated by the isolated BBM according to the status of tubular phosphate reabsorption presented in vivo, but no clear mechanism responsible for this "memory" has yet been identified. The mechanisms involved in the transport of phosphate through the cytosol are very poorly understood. The role of the concentration of the oxidized form of NAD remains controversial. Intracellular calcium is probably an important factor in phosphate tubular transport, possibly through its inhibitory effect upon the adenylate cyclase activity, which is an important step in the cascade of events initiated by PTH binding to the basolateral membrane. Finally the $1,25(\mathrm{OH})_{2} \mathrm{D}_{3}$ which is synthesized in the proximal tubule cell, also regulates phosphate transport, possibly through a modification of the intracellular phosphate crosses the basolateral membrane passively, along with a positive electrical potential. The main role of this membrane in phosphate transport could then be to carry the receptors of a variety of hormones, and particularly the parathyroid hormone, with the systems of regulatory protein and enzymes which are under its dependency. calcium concentration.

It is generally believed that 